\title{
Texture Based Mammogram Registration Using Geodesic Interpolating Splines
}

\author{
Styliani Petroudi and Michael Brady \\ Medical Vision Laboratory, Oxford University, Oxford, OX2 7DD, United Kingdom \\ \{styliani, jmb\}@robots.ox.ac.uk
}

\begin{abstract}
We propose a new approach for nonrigid registration of mammograms. We use texture models for the mammographic appearance within the breast area. The mammograms are registered, based on corresponding points along the boundaries of the region of adipose tissue near the breast edge, using geodesic interpolating splines.
\end{abstract}

\section{Introduction}

Registration of mammograms is required for analysis of bilateral, temporal, and different view mammograms, and for multi-subject studies. Mammogram registration has proven a difficult problem, not least because of the enormous diversity in the appearance of mammograms. Various classes of image transformations for registration have been investigated, including flow and thin plate splines. Recent progress in the estimation of flows of diffeomorphisms have resulted in the development of geodesic interpolating splines (GIS) [1]. These provide an unambiguous one-to-one correspondence between all points in any pair of images. Registration results can be used to detect changes in the breast tissue that are characteristic of cancer. Most algorithms compare registered image intensities or a representation derived from image intensities, which requires some form of normalization. However, we have shown that it is possible to compute a representation of textures within a mammogram; these are known to be significant in the evaluation of disease.

\section{Method and Results}

In this paper we propose an approach to matching mammograms which combines texture classes as image descriptors and GIS. Texture classes are used to establish control points for the geodesic warps and to evaluate the outcomes for temporal matching. The preliminary results are very encouraging. Textons are defined as clustered filter responses, where the set of filters used, mirrors a non-parametric representation of Markov Random Fields. First we construct a texton dictionary by processing a large number of segmented mammograms and then aggregating and clustering filter responses using k-means analysis. Given the texton dictionary, each image pixel in the breast region is assigned a label by the texton which lies closest to it in the filter space. A texture based classifier 
segments the image in different tissue types of varying parenchymal density as in Figure 1(d) and (e). One of these classes corresponds to the fatty breast edge which can be consistently observed in all the mammograms. The boundary of the transition from the fatty breast edge to dense tissue, along with the nipple, the axilla and the rib, and other points on the breast boundary provide the control points. After mapping the points in both views onto each other the warped image is obtained by interpolating between the control points using geodesic interpolating splines. The GIS uses, instead of one large step, a succession of small diffeomorphic steps to warp the image [2]. A pair of registered mammograms is shown in Figure 1(a),(b) and (c). To compare the registered mammograms, the warped image is also segmented into the different tissue types, using the presented texture model followed by a texture-based classifier. A region is labelled suspicious if it corresponds to tissue that has evolved to be very dense. We are still developing a more robust system for evaluating the tissue changes. The complete algorithm has so far been applied to 10 pairs of temporal mammograms with excellent results.

a.

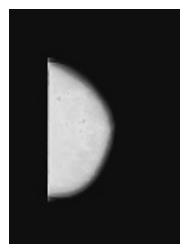

b.

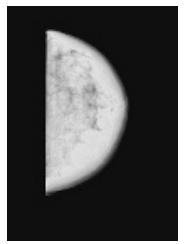

c.

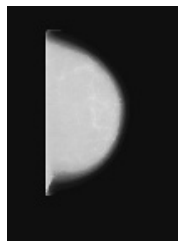

d.

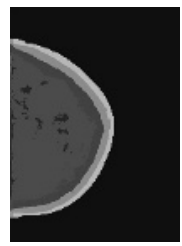

e.

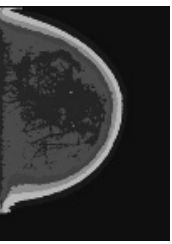

Fig. 1. (a)The mammogram that needs registration. (b)The base mammogram. (c)The geodesically registered mammogram. (d-e)The texture based representation of (a-b) respectively.

\section{Conclusions}

We have presented an algorithm for nonrigid mammogram registration which investigates the combination of texture models with GIS. The use of texture models provides a reliable framework for establishing correspondences and detecting comparative changes in mammograms. The method overcomes the difficulty arising form the degree of involution in the breast tissue.

\section{References}

1. V. Camion and L. Younes. Geodesic interpolating splines. In Proceedings of EMMCVPR'01, volume 2134, pages 513-527, 2001.

2. C. J. Twining and S. Marsland. Constructing diffeomorphic representations of nonrigid registrations of medical images. In IPMI 2003, 2003. 